\author{
DEPARTMENT OF THE INTERIOR \\ UNITED STATES GEOLOGICAL SURVEY
}

\title{
Preliminary Data Report conducted for the Colorado State Geological Survey on the Superconducting, Supercollider Study
}

By

Donley S. Collins ${ }^{1}$ and Thomas C. Nichols, Jr. ${ }^{1}$

This report is preliminary and has not been reviewed for conformity with U.S. Geological Survey editorial standards and stratigraphic nomenclature. Any use of trade names is for descriptive purposes only and does not imply endorsement by the USGS.

Open-File Report 87-55

1U.S. Geological Survey

Denver, Colorado 


\section{CONTENTS}

Page

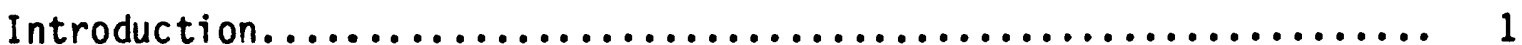

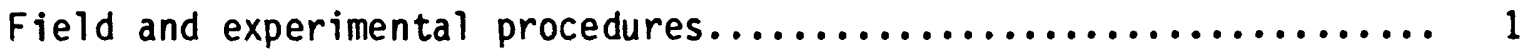

Summary of the principal rock types and their geotechnical

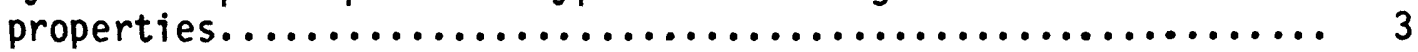

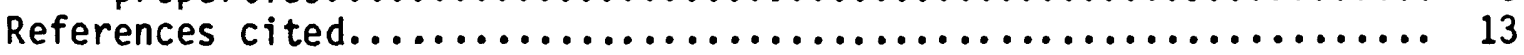

\section{ILLUSTRATIONS}

Figure 1. Location of CSGS drill hole 10 (dot and arrow)........ 2

2. Graphic representation of depth versus

moisture content. Open circte represents

an average value of three moisture tests.

Arrow indicates the location of the only

confirmed fracture within logged core.

$X$ indicates position of possible gouge

material............................... 12

TABLE

Table 1. Selected moisture content and bulk density determinations, natural fracture, and generalized lithologic descriptions of core from the CSGS drill hole $10 \ldots . . \ldots \ldots . . . . . .$. 


\section{Prelininary Data Report Conducted for the \\ Colorado State Geological Survey on the Superconducting, Supercollider Study}

Donley S. Collins and Thomas C. Nichols, Jr.

\section{INTRODUCTION}

This report documents the geological and geotechnical data acquired from one drill hole that is part of Colorado's SSC (Superconducting Supercollider) site study initiated to consider the Cretacous Pierre Shale as a host material for the SSC facility. Upon request from the CSGS (Colorado State Geological Survey), the USGS (U.S. Geological Survey) participated in this study by gathering and compiling geologic and geotechnical data from core retrieved from CSGS drill hole 10 .

The drill site, ground elevation $4,750 \mathrm{ft}$, is located in the NW1/4, Sec. 17, R. 57 W., T. 2 S. (U.S. Geological Survey, 1976), near Last Chance, Colorado (fig. 1.), 75 mi east of Denver, Colorado. In March 1986, drilling began in soil and penetrated and reached total depth within the Pierre Shale (Late Cretaceous age).

\section{FIELD NDD EXPERIMENTAL PROCEDURES}

The hole was drilled and core was recovered by using a truck-mounted mobile B-80 auger/core drill. The first $20 \mathrm{ft}$ from the surface were augered with a 4-in.-outside-diameter auger pipe that was left in the hole and used as surface casing for coring. At a depth of $20 \mathrm{ft}$, when the hardness of the formational material exceeded 50 blows per foot by a 140-1b hammer through a 30-in. drop on a California sampler, the material was sufficiently hard to core. A Christensen NX-size wire-line with a split inner barrel and tungstencarbide bit was used to advance the hole to a total depth of $277 \mathrm{ft}$. Core samples, having an average diameter of 1.78 in., were obtained with split-tube inner core barrels as much as $10 \mathrm{ft}$ in length. Core recovery was greater than 95 percent. The hole was advanced using only water as the circulating drill fluid.

In the field, the core was removed from the split inner barrel of the core barrel, logged, and tested for carbonate content with 10 percent solution of HCL. Concurrently, a rock-color chart (Rock-color chart committee, 1975) was used for core-color description. After logging, selected core samples were used for as-received bulk density tests and moisture content determinations. Three as-received bulk densities were determined for each sample in a manner described by Chleborad and others (1975), with the following exception: the samples were not oven dried prior to waxing. Similarly, to determine moisture content, three subsamples for each selected sample were weighed and placed in preweighed tins immediately after logging. These samples were later transported to the USGS Laboratory in Denver, Colorado, where they were oven dried for $24 \mathrm{hrs}$ at a constant temperature of $110^{\circ} \mathrm{C}$ and reweighed. 

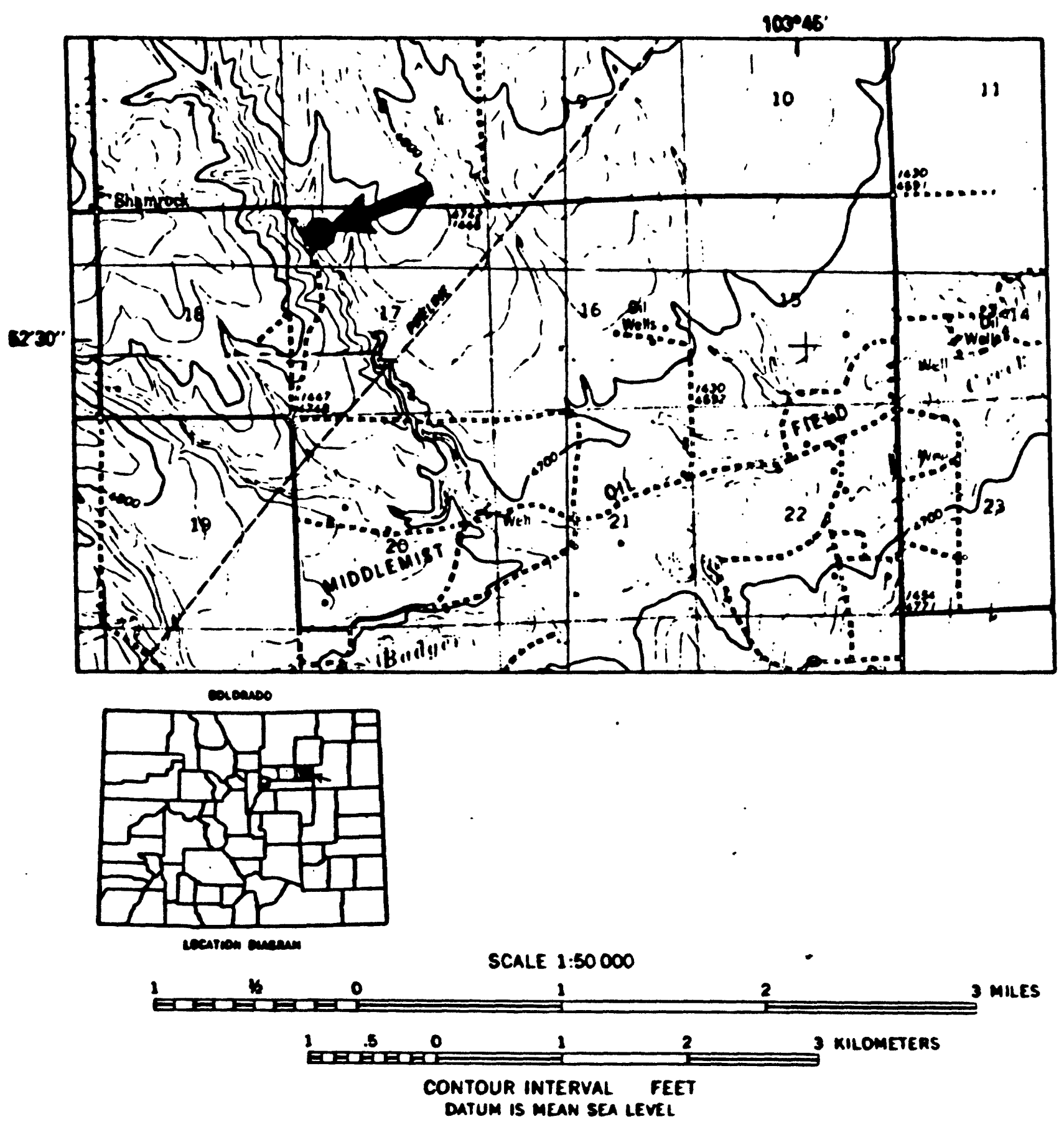

Figure 1. Location of CSGS drill hole 10 (dot and arrow). 


\section{SUMMARY OF THE PRINCIPAL ROCK TYPES AND THEIR GEOTECHNICAL CHARACTERISTICS}

Table 1 summarizes the lithology, natural fractures, and geotechnical properties measured on selected core samples. The average value for each set of three samples is presented in table 1 for both the as-received bulk density and the moisture content.

The upper $20 \mathrm{ft}$ of the section consist of weak surficial materials and weathered shale that were sampled by auger (Mark Davis, CSGS geologist, oral commun., 1986), but not logged or analyzed in this part of the study. Underlying this material and extending to a depth of $68.5 \mathrm{ft}$, the lithology is primarily a non- to very silty, noncalcareous claystone. Beginning about 36 $\mathrm{ft}$, moisture content is 0.82 weight percent lower than that at $24 \mathrm{ft}$. However, from the 36-ft level downward, there is a slight increase in moisture content, and a continued slight decrease in bulk density for the 20-68.5-ft interval.

From 68.5 to $108.0 \mathrm{ft}$, the sediment consists predominantly of claystone that varies from slightly silty to silty. Within this interval, there is a continued silght increase in moisture content from 15.08 to 16.44 percent and in bulk density from 2.06 to about 2.12 . The last $39.5 \mathrm{ft}$ of claystone material show a moderate reaction to $\mathrm{HCl}$. Al so present within this interval is a limestone bed $0.8 \mathrm{ft}$ thick at $84.0 \mathrm{ft}$, and four bentonite beds present between 93.4-95.8 ft that vary in thickness from 0.5 to 1 in. One fracture, having a dip of $55^{\circ}$, was observed at $106.4 \mathrm{ft}$. Based on our limited moisture data, this fracture appears to be within a "zone" (from 88.0 to $134.5 \mathrm{ft}$ ) of higher moisture content (fig. 2 ).

From 108.0 to $239.0 \mathrm{ft}$, the claystone varies from shaley to silty and is primarily noncalcareous except for a limy zone between 209.9 and $210.1 \mathrm{ft}$. Silt lamina are common with in this interval; and silt bedding are only present below $209.0 \mathrm{ft}$. Moisture content decreases slightly from about 15.46 to 13.78, whereas, bulk density varies only by 0.001 . Limestone beds having thicknesses of 0.8 and $0.4 \mathrm{ft}$ were found at 149.4 and $182.35 \mathrm{ft}$, respectively. A possible "gouge zone" is present near the 113.0 depth and lies just below the fracture at $106.4 \mathrm{ft}$ and within the high moisture "zone" (fig. 2).

The last interva1, 239.0-277.0 ft, has three zones starting at 239.0, 249.0 , and $259.0 \mathrm{ft}$ in which claystone appears to grade downward into a clayey siltstone. Limestone beds are present at 242.6 and $269.0 \mathrm{ft}$ with thicknesses of 0.80 and $3.0 \mathrm{ft}$, respectively. Only one moisture content $(15.16$ weight percent) and one bulk density (2.16) were determined within this interval. The apparent higher moisture content within this interval, as compared to that found within the 108.0-239.0-ft interval above (fig. 2), probably corresponds to the increase in silt content and silt lamina. 
TABLE 1. Selected moisture and bulk density determinations, natural fracture, and generalized lithologic descriptions of core from the Colorado State Geological Survey drill hole 10

[--- indicates information is unavailable]

\begin{tabular}{|c|c|c|c|c|c|c|}
\hline $\begin{array}{l}\text { Depth } \\
\text { in tenths } \\
\text { of } \mathrm{ft}\end{array}$ & Description & Color & $\begin{array}{l}\text { Natural } \\
\text { fracture } \\
\text { descrip- } \\
\text { tion }\end{array}$ & $\begin{array}{l}\text { Depth } \\
\text { of } \\
\text { sample } \\
(f t)\end{array}$ & $\begin{array}{l}\text { Moisture } \\
\text { content } \\
\text { (pct. wt) }\end{array}$ & $\begin{array}{l}\text { Bulk } \\
\text { density } \\
\text { (gm/cc) }\end{array}$ \\
\hline $20.0-21.3$ & $\begin{array}{l}\text { Claystone, } \\
\text { silty......... }\end{array}$ & $\begin{array}{l}\text { Medium dark } \\
\text { gray (N3). }\end{array}$ & None. & & & \\
\hline $21.3-25.0$ & $\begin{array}{l}\text { Claystone, } \\
\text { very silty, } \\
\text { noncalcar- } \\
\text { eous......... }\end{array}$ & $\begin{array}{l}\text { Medium gray } \\
\text { to medium } \\
\text { dark gray } \\
\text { (N3-N4). }\end{array}$ & --do---- & 24.0 & 15.85 & 2.13 \\
\hline $25.0-36.6$ & $\begin{array}{l}\text { Claystone, } \\
\text { noncalcar- } \\
\text { eous......... }\end{array}$ & -- do--.-.--- & --do---- & 36.0 & 15.03 & 2.12 \\
\hline $36.6-40.0$ & $\begin{array}{l}\text { Claystone, } \\
\text { very slight- } \\
\text { ly silty } \\
\text { to non- } \\
\text { silty, non- } \\
\text { calcareous... }\end{array}$ & -- do------.-- & -- do---- & --- & -- & -- \\
\hline $40.0-48.0$ & $\begin{array}{l}\text { Claystone, } \\
\text { very, } \\
\text { slightly } \\
\text { silty to } \\
\text { non-silty, } \\
\text { noncalcar- } \\
\text { eous......... }\end{array}$ & -- do-- & --do---- & 44.0 & 15.05 & 2.11 \\
\hline $48.0-58.5$ & $\begin{array}{l}\text { Claystone, } \\
\text { slightly } \\
\text { silty, non- } \\
\text { calcareous... }\end{array}$ & --do--.--...-- & -- do---- &.-- & --- & $\ldots$ \\
\hline $58.5-68.5$ & $\begin{array}{l}\text { Claystone, } \\
\text { very slight- } \\
\text { ly silty, } \\
\text { noncalcareous. } \\
\text { At } 62.7 \mathrm{ft} \\
\text { bentonite bed } \\
1 \text { in. thick.. }\end{array}$ & --do--------- & -- do---- & 61.9 & 15.08 & 2.06 \\
\hline
\end{tabular}


TABLE 1. Selected moisture and bulk density determinations, natural fracture, and generalized 1 ithologic descriptions of core from the Colorado State Geological Survey drill hole 10--Continued

[.-- indicates information is unavailable]

\begin{tabular}{|c|c|c|c|c|c|c|}
\hline $\begin{array}{c}\text { Depth } \\
\text { in tenths } \\
\text { of } \mathrm{ft}\end{array}$ & Description & Color & $\begin{array}{l}\text { Natural } \\
\text { fracture } \\
\text { descrip- } \\
\quad \text { tion }\end{array}$ & $\begin{array}{l}\text { Depth } \\
\text { of } \\
\text { sample } \\
(\mathrm{ft})\end{array}$ & $\begin{array}{c}\text { Moisture }{ }^{2} \\
\text { content } \\
\text { (pct. wt) }\end{array}$ & $\begin{array}{l}\text { Bulk } \\
\text { density } \\
(g \mathrm{~m} / \mathrm{cc})\end{array}$ \\
\hline $68.5-78.5$ & $\begin{array}{l}\text { Claystone, } \\
\text { silty, noncal- } \\
\text { careous, burrows } \\
\text { and one Pelecyp } \\
\text { valve......... }\end{array}$ & $\begin{array}{l}\text { Medium grey } \\
\text { (N4). } \\
\text { ws } \\
\text { pod }\end{array}$ & --do--.- & -- & -- &.-- \\
\hline $78.5-88.5$ & $\begin{array}{l}\text { Claystone, } \\
\text { silty, noncal- } \\
\text { careous. Lime- } \\
\text { stone } 84.0- \\
84.8 \mathrm{ft} . . . .\end{array}$ & $\begin{array}{l}\text { Medium gray } \\
\text { (claystone) } \\
\text { (N4); light } \\
\text { gray (lime- } \\
\text { stone) (N4). }\end{array}$ & --do---- & 88.0 & 15.12 & 2.13 \\
\hline $88.5-98.5$ & $\begin{array}{l}\text { Claystone, } \\
\text { silty, noncal- } \\
\text { careous. Four } \\
\text { bentonite beds: } \\
\text { (1) } 1 \text { in. thick } \\
\text { at } 93.4 \mathrm{ft} \text { ( } 2) \\
1 \text { in. thick at } \\
94.4 \mathrm{ft} ;(3) \\
1 / 2 \mathrm{in} \text {. thick at } \\
94.9 \mathrm{ft} ;(4) 1 \\
\text { in. thick at } \\
95.8 \mathrm{ft} . . . . .\end{array}$ & $\begin{array}{l}\text { Medium grey } \\
(N 4) \text {. } \\
\text { at }\end{array}$ & -- do---- & --- & --- & --- \\
\hline $98.5-108.0$ & $\begin{array}{l}\text { Claystone, } \\
\text { slightly silty, } \\
\text { noncalcareous } \\
\text { to moderately } \\
\text { calcareous, } \\
\text { burrows and } \\
\text { one coiled } \\
\text { Ammonite } \\
\text { present...... }\end{array}$ & - do- & $\begin{array}{l}\text { One frac- } \\
\text { ture at } \\
106.4 \mathrm{ft} . \\
\text { Flat frac } \\
\text { ture surf } \\
\text { with a } 55 \\
\text { Slicks nc } \\
\text { visible c } \\
\text { to mud } \\
\text { coating. }\end{array}$ & $\begin{array}{l}100.0 \\
\text { c- } \\
\text { face } \\
5^{\circ} \text { dip. } \\
\text { ot } \\
\text { due }\end{array}$ & 16.44 & 2.12 \\
\hline
\end{tabular}


TABLE 1. Selected moisture and bulk density determinations, natural fracture, and generalized lithologic descriptions of core from the Colorado State Geological Survey drill hole 10--Continued

[--- indicates information is unavailable]

\begin{tabular}{|c|c|c|c|c|c|c|}
\hline $\begin{array}{l}\text { Depth } \\
\text { in tenths } \\
\text { of } \mathrm{ft}\end{array}$ & Description & Color & $\begin{array}{l}\text { Natural } \\
\text { fracture } \\
\text { descrip- } \\
\text { tion }\end{array}$ & $\begin{array}{l}\text { Depth } \\
\text { of } \\
\text { sample } \\
(f t)\end{array}$ & $\begin{array}{l}\text { Moisture } \\
\text { content } \\
\text { (pct. wt) }\end{array}$ & $\begin{array}{l}\text { Bulk } \\
\text { density } \\
\text { (gm/cc) }\end{array}$ \\
\hline $108.0-110.7$ & $\begin{array}{l}\text { Claystone, } \\
\text { silty, non- } \\
\text { calcareous... }\end{array}$ & --do---- & None & --- & --- & --- \\
\hline $110.7-113.0$ & $\begin{array}{l}\text { Claystone, } \\
\text { slightly silty, } \\
\text { noncalcareous. }\end{array}$ & -- do--.---.--- & $\begin{array}{l}\text { Lower part } \\
\text { of core } \\
\text { could be } \\
\text { gouge(?). }\end{array}$ & -.- & --- & --- \\
\hline $113.0-120.0$ & $\begin{array}{l}\text { Claystone, } \\
\text { slightly } \\
\text { silty, non- } \\
\text { calcareous... }\end{array}$ & $\begin{array}{l}\text { Medium light } \\
\text { gray to me- } \\
\text { dium gray } \\
\text { (N5-N6). }\end{array}$ & None. & 115.5 & 15.46 & 2.13 \\
\hline $120.0-1303$ & $\begin{array}{l}\text { Claystone, } \\
\text { shal ey, non- } \\
\text { calcareous... }\end{array}$ & $\begin{array}{r}\text { Medium dark } \\
\text { gray }(N 3) .\end{array}$ & --do---- &.-- &.-- & --- \\
\hline $130.3-140.0$ & $\begin{array}{l}\text { Claystone, } \\
\text { silty, shaley } \\
\text { with inter- } \\
\text { bedded silt } \\
\text { lamina........ }\end{array}$ & - do-- - - & --do---- & 134.5 & 14.70 & 2.15 \\
\hline $140.0-149.5$ & $\begin{array}{l}\text { Claystone, } \\
\text { silty, very, } \\
\text { slightly } \\
\text { calcareous } \\
\text { to noncalcar- } \\
\text { eous. Coiled } \\
\text { Ammonite at } \\
137.8 \mathrm{ft} \text { and } \\
\text { vertical burrow } \\
\text { present...... }\end{array}$ & $\begin{array}{l}\text { Medium light } \\
\text { gray to medi- } \\
\text { um gray } \\
(\text { N5-N4). } \\
\text { s }\end{array}$ & --do---- & $144.6^{\circ}$ & 14.49 & 2.14 \\
\hline
\end{tabular}


TABLE 1. Selected moisture and bulk density determinations, natural fracture, and generalized lithologic descriptions of core from the Colorado State Geological Survey drill hole 10--Continued

[-.- indicates information is unavailable]

\begin{tabular}{|c|c|c|c|c|c|c|}
\hline $\begin{array}{l}\text { Depth } \\
\text { in tenths } \\
\text { of } \mathrm{ft}\end{array}$ & Description & Color & $\begin{array}{l}\text { Natural } \\
\text { fracture } \\
\text { descrip- } \\
\text { tion }\end{array}$ & $\begin{array}{l}\text { Depth } \\
\text { of } \\
\text { sample } \\
(\mathrm{ft})\end{array}$ & $\begin{array}{c}\text { Moisture } \\
\text { content } \\
\text { (pct. wt) }\end{array}$ & $\begin{array}{l}\text { Bulk } \\
\text { density } \\
\text { (gm/cc) }\end{array}$ \\
\hline $149.5-159.0$ & $\begin{array}{l}\text { Limestone, } \\
149.4-150.2 \\
\text { ft; clay- } \\
\text { stone, shaley, } \\
\text { silty } 150.2- \\
151.2 \mathrm{ft} ; \\
\text { claystone, } \\
\text { silty } 151.2- \\
159.0 \mathrm{ft} . . . .\end{array}$ & $\begin{array}{c}\text { Medium } 1 \text { ight } \\
\text { gray (N5). }\end{array}$ & -- do---- & -.- & --- &.-- \\
\hline $159.0-169.0$ & $\begin{array}{l}\text { Claystone, } \\
\text { silty, non- } \\
\text { calcareous } \\
\text { with discon- } \\
\text { tinuous inter- } \\
\text { bedded silt } \\
\text { lamina and } \\
\text { beds. Hori- } \\
\text { zontal burrows } \\
\text { present...... }\end{array}$ & $\begin{array}{l}\text { Medium dark } \\
\text { gray (N3). }\end{array}$ & -- do-- - - &.-- & -- & -.- \\
\hline $169.0-179.0$ & $\begin{array}{l}\text { Claystone, } \\
\text { silty, non- } \\
\text { calcareous. } \\
\text { Silt content } \\
\text { increases down- } \\
\text { ward. Silt } \\
\text { lamina increas- } \\
\text { es downward. } \\
\text { Pelecypod at } \\
172.6 \mathrm{ft} . . . .\end{array}$ & $\begin{array}{l}\text { Medium gray } \\
\text { (N4). }\end{array}$ & --do----.-- & 170.5 & 14.33 & 2.14 \\
\hline
\end{tabular}


TABLE 1. Selected moisture and bulk density determinations, natural fracture, and generalized lithologic descriptions of core from the Colorado State Geological Survey drill hole 10--Continued

[-.- indicates information is unavailable]

\begin{tabular}{|c|c|c|c|c|c|c|}
\hline $\begin{array}{l}\text { Depth } \\
\text { in tenths } \\
\text { of } \mathrm{ft}\end{array}$ & Description & Color & $\begin{array}{l}\text { Natural } \\
\text { fracture } \\
\text { descrip- } \\
\text { tion }\end{array}$ & $\begin{array}{c}\text { Depth } \\
\text { of } \\
\text { sample } \\
(\mathrm{ft})\end{array}$ & $\begin{array}{c}\text { Moisture }{ }^{2} \\
\text { content } \\
\text { (pct. wt) }\end{array}$ & $\begin{array}{l}\text { Bulk }{ }^{2} \\
\text { density } \\
(g m / c c)\end{array}$ \\
\hline $179.0-189.0$ & $\begin{array}{l}\text { Claystone, } \\
\text { silty } 179.0- \\
182.35 \mathrm{ft} ; \\
\text { limestone } \\
182.35-182.75 \\
\text { ft; claystone } \\
\text { shaley } 182.75- \\
189.0 \mathrm{ft} . . . .\end{array}$ & $\begin{array}{l}\text { Very light gray } \\
\text { to white (lime- } \\
\text { stone) (N7-N8) } \\
\text { medium dark } \\
\text { gray (clay- } \\
\text { stone) (N3). }\end{array}$ & ) &.-- &.- & -- \\
\hline $189.0-199.3$ & $\begin{array}{l}\text { Claystone, } \\
\text { silty, non- } \\
\text { calcareous. } \\
\text { Becomes shaley } \\
\text { and moderately } \\
\text { calcareous } \\
198.4-199.3 \mathrm{ft} \text {. }\end{array}$ & $\begin{array}{l}\text { Medium gray } \\
\text { (N4). }\end{array}$ & -- do $-\cdots$ & 195.5 & 14.37 & 2.15 \\
\hline $199.3-209.0$ & $\begin{array}{l}\text { Claystone, } \\
\text { silty with } \\
\text { discontin- } \\
\text { uous silt } \\
\text { lamina } \\
199.3-202.3 \\
\text { ft; claystone, } \\
\text { shaley } 202.3- \\
203.3 \mathrm{ft} ; \\
\text { claystone, } \\
\text { silty with dis- } \\
\text { continuous silt } \\
\text { lamina } 203.2- \\
\text { 209.0 ft. } \\
\text { Whole interval } \\
\text { slightly to non } \\
\text { calcareous. Ho } \\
\text { zontal burrows } \\
\text { present through. } \\
\text { out........... }\end{array}$ & - & -- do.--- & -- &.- & -.. \\
\hline
\end{tabular}


TABLE 1. Selected moisture and bulk density determinations, natural fracture, and generalized lithologic descriptions of core from the Colorado State Geological Survey drill hole 10--Continued

[--- indicates information is unavailable]

\begin{tabular}{|c|c|c|c|c|c|c|}
\hline $\begin{array}{l}\text { Depth } \\
\text { in tenths } \\
\text { of } \mathrm{ft}\end{array}$ & Description & Color & $\begin{array}{l}\text { Natural } \\
\text { fracture } \\
\text { descrip- } \\
\text { tion }\end{array}$ & $\begin{array}{l}\text { Depth } \\
\text { of } \\
\text { samp le } \\
(\mathrm{ft})\end{array}$ & $\begin{array}{c}\text { Moisture } \\
\text { content } \\
\text { (pct. wt) }\end{array}$ & $\begin{array}{l}\text { Bulk } \\
\text { density } \\
\text { (gm/cc) }\end{array}$ \\
\hline $209.0-219.0$ & $\begin{array}{l}\text { Claystone, } \\
\text { silty, non- } \\
\text { calcareous. } \\
\text { silt lamina } \\
\text { and bedding } \\
\text { throughout. } \\
\text { Horizontal } \\
\text { burrows present } \\
\text { A limy zone } \\
209.9-210.1 \mathrm{ft} .\end{array}$ & $\begin{array}{l}\text { Medium aray to } \\
\text { medium dark } \\
\text { gray (N4-N3). } \\
\text { t. }\end{array}$ & -- do---- & 215.0 & 13.74 & 2.17 \\
\hline $219.0-229.0$ & $\begin{array}{l}\text { Claystone, } \\
\text { very, } \\
\text { slightly } \\
\text { silty to } \\
\text { non-silty, } \\
\text { noncalcar- } \\
\text { eous } 219.0- \\
228.8 \mathrm{ft} \text {; } \\
\text { Claystone, } \\
\text { silty, noncal- } \\
\text { careous with } \\
\text { silt beds and } \\
\text { lamina } 228.8- \\
229.0 \mathrm{ft} . . . .\end{array}$ & Dark gray (N2). & -- do---- & -- & -- & --- \\
\hline $229.0-239.0$ & $\begin{array}{l}\text { Claystone, } \\
\text { silty, inter- } \\
\text { bedded silt- } \\
\text { stone, moder- } \\
\text { ately calcar- } \\
\text { eous. Hori- } \\
\text { zontal and } \\
\text { vertical } \\
\text { burrows } \\
\text { present...... }\end{array}$ & $\begin{array}{l}\text { Medium dark } \\
\text { gray (silty } \\
\text { Claystone) } \\
\text { (N3), Medium } \\
\text { light gray } \\
\text { (siltstone) } \\
\text { (N6). }\end{array}$ & --do---- & 229.5 & 13.78 & 2.18 \\
\hline
\end{tabular}


TABLE 1. Selected moisture and bulk density determinations, natural fracture, and generalized lithologic descriptions of core from the Colorado State Geological Survey drill hole 10--Continued

[--- indicates information is unavailable]

\begin{tabular}{|c|c|c|c|c|c|c|}
\hline $\begin{array}{l}\text { Depth } \\
\text { in tenths } \\
\text { of } \mathrm{ft}\end{array}$ & Description & Color & $\begin{array}{l}\text { Natural } \\
\text { fracture } \\
\text { descrip- } \\
\text { tion }\end{array}$ & $\begin{array}{l}\text { Depth } \\
\text { of } \\
\text { sample } \\
(\mathrm{ft})\end{array}$ & $\begin{array}{c}\text { Moisture } \\
\text { content } \\
\text { (pct. wt) }\end{array}$ & $\begin{array}{l}\text { Bulk } \\
\text { density } \\
(g m / c c)\end{array}$ \\
\hline $239.0-249.0$ & $\begin{array}{l}\text { Claystone, } \\
\text { silty changing } \\
\text { to siltstone, } \\
\text { clayey, non- } \\
\text { calcareous with } \\
\text { silt lamina. } \\
\text { Limestone, vugg } \\
242.6-243.4 \mathrm{ft} \text {. }\end{array}$ & $\begin{array}{l}\text { Medium gray } \\
\text { (claystone } \\
\text { and clayey } \\
\text { siltstone) } \\
\text { (N4). }\end{array}$ & --do----- & --- & --- & --- \\
\hline $249.0-259.0$ & $\begin{array}{l}\text { Claystone, } \\
\text { silty changing } \\
\text { to siltstone, } \\
\text { clayey, non- } \\
\text { calcareous. } \\
\text { Burrows and } \\
\text { brown pelecypod } \\
\text { shell fragment } \\
\text { present...... }\end{array}$ & $\begin{array}{l}\text { Medium light } \\
\text { gray (clayey } \\
\text { siltstone) } \\
\text { (N5); medium } \\
\text { dark gray } \\
\text { (silty } \\
\text { claystone) } \\
\text { (N3). }\end{array}$ & -- do---- & 251.5 & 15.16 & 2.16 \\
\hline $259.0-269.0$ & $\begin{array}{l}\text { Claystone, } \\
\text { silty, non- } \\
\text { calcareous. } \\
\text { Increase in } \\
\text { silt downward } \\
\text { to become silt- } \\
\text { stone, clayey, } \\
\text { noncalcareoue. } \\
\text { Calcareous } \\
268.8-268.85 \\
\text { ft. Horizon- } \\
\text { tal burrows } \\
\text { present...... }\end{array}$ & $\begin{array}{l}\text { Medium gray to } \\
\text { dark gray } \\
\text { (N4-N3). }\end{array}$ & -- do----- & --- & -- & -- \\
\hline
\end{tabular}


TABLE 1. Selected moisture and bulk density determinations, natural fracture, and generalized lithologic descriptions of core from the Colorado State Geological Survey drill hole 10--Continued

$$
\text { [--- indicates information is unavailable] }
$$

\begin{tabular}{|c|c|c|c|c|c|c|}
\hline $\begin{array}{l}\text { Depth } \\
\text { in tenths } \\
\text { of } \mathrm{ft}\end{array}$ & Description & Color & $\begin{array}{l}\text { Natural } \\
\text { fracture } \\
\text { descrip- } \\
\text { tion }\end{array}$ & $\begin{array}{l}\text { Depth } \\
\text { of } \\
\text { sample } \\
(\mathrm{ft})\end{array}$ & $\begin{array}{l}\text { Moisture } \\
\text { content } \\
\text { (pct. wt) }\end{array}$ & $\begin{array}{l}\text { Bulk } \\
\text { density } \\
(g m / c c)\end{array}$ \\
\hline $269-277.0$ & $\begin{array}{l}\text { Claystone, } \\
\text { silty; iime- } \\
\text { stone } 269.0- \\
272.0 \mathrm{ft} . . . .\end{array}$ & $\begin{array}{l}\text { Medium dark } \\
\text { gray (clay- } \\
\text { stone) light } \\
\text { gray to white } \\
\text { (limestone) } \\
\text { (N7-N8). }\end{array}$ & --do---- & -- & -- & $\ldots$ \\
\hline
\end{tabular}

1 Approximate depth $(\mathrm{ft})$ of sample used for moisture content and bulk density determinations.

2

Value represents average of three tests. 


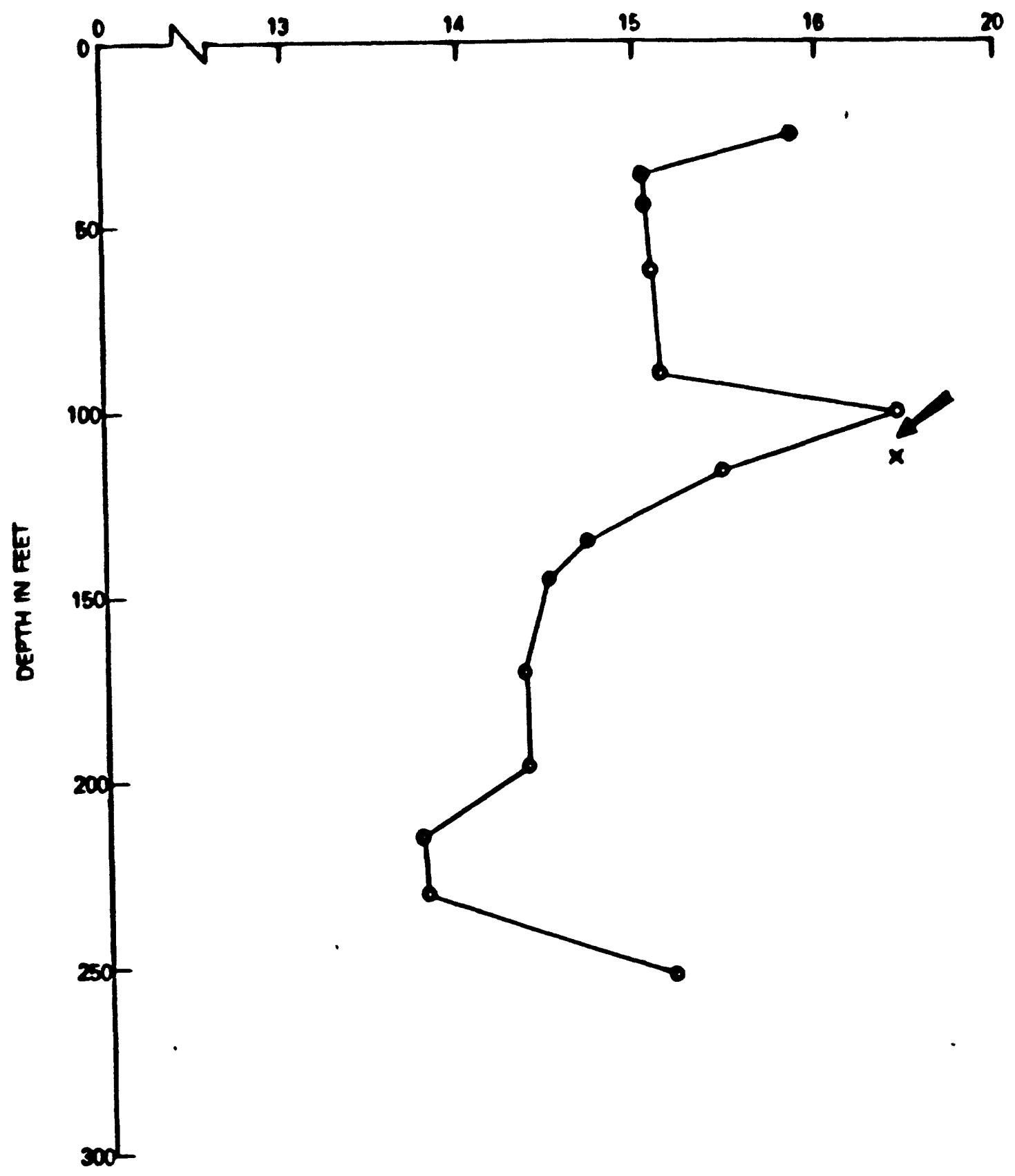

Figure 2. Graphic representation of depth versus moisture content. Open circle represents an average value of three moisture tests. Arrow indicates the location of the only confirmed fracture within logged core. $X$ indicates position of possible gouge material. 


\section{REFERENCES CITED}

1. Chleborad, A.F., Powers, P.S., and Farrow, R.A., 1975, A technique for measuring bulk volume of rock materials: Association of Engineering Geologists Bulletin, v. 12, no. 4, p. 317-322.

2. Rock-color Chart, 1975, The Geological Society of America, Boulder, Colorado, $10 \mathrm{p}$.

3. U.S. Geological Survey, 1976, Adams County, Colorado, topographic map: U.S. Geological Survey, scale 1:50,000, sheet 3 . 